\title{
SEROTHERAPY OF EPIDEMIC CEREBROSPINAL MENINGITIS.
}

REPORT OH TIVELVE CASES.

W. S. CHASF, M.D., ANV M. T. I[CX]. M.1).

AKRON, OHIO.

In the months of April, May and June, 1907, there were reported to the Health Officer of Akron 22 cases of epidemic cerebrospinal meningitis. The first case came down April 28, the most of them occurring in May and June. The last one was admitted to the hospital September 11, having first shown symptoms six days before.

Four of the patients, strong, healthy young men, were employed at one of the local rubber factories. In no other instance could there be shown any possibility of communication by contact. In fact, a striking feature of the epidemic was the occurrence, at the same time, in widely separated districts of the city, of single cases.

Twelve of the cases were treated at the Akron City Hospital. Of these, 3 of the patients died and 9 recovered, a mortality of 25 per cent. Of the cases outside, one patient recovered and 9 died, a mortality of 90 per cent. All of those treated at the hospital received the Flexner antiserum. Those treated outside did not. The diagnosis of the case of the one outside patient, who recovered, was not confirmed by lumbar puncture and presented atypical symptoms.

While realizing the ease with which the post hoc may blossom into the propter hoc, when the treatment of any disease is concerned, it seems to us that the striking difference in the mortality rate of these two sets of cases is more than enough to warrant a further and wider trial of the Flexner antiserum.

At the time of the outbreak in Akron the Flexner antiserum had been tried in a few cases at Castalia, Ohio. A supply of this serum was sent to Akron by Dr. Flexner from the Rockefeller Institute of New York, with the understanding that the necessary bacteriologic examinations be made in each case. In order that this might be done under the most favorable conditions, it was decided that the serum should be administered only to patients received at the Akron City Hospital. That fact was communicated to the physicians of the city, and a committee of three physicians from the medical staff of the hospital was appointed to see in consultation the cases of epidemic spinal meningitis admitted, and to have general supervision of the serum administration and of the bac- 
teriologic work. That committee consisted of Drs. A. A. Kohler, C. E. Held, and W. S. Chase. They were assisted in their work by Drs. M. I. Hunt and G. M. Logan, the hospital internes.

The diagnosis was made in no case on the clinical signs alone, but these were supplemented, in each instance, by microscopic and culture tests of the spinal fluid withdrawn by lumbar puncture. A puncture was done under aseptic precautions whenever clinical evidence warranted it and as soon as possible after the patient's admission.

In the first few cases the antiserum was not administered until after a microscopic examination of the withdrawn fluid had been made showing the presence of Weichselbaum's Diplococcus intracellularis. Later on, when the clinical evidence seemed positive, the injection of the serum was made at the time of the first puncture; but in all cases the clinical evidence was corroborated by the subsequent microscopic findings.

As all the patients were more or less wildly delirious, it was found advisable to perform lumbar puncture, in almost every instance, under general anesthesia. For that purpose chloroform was used. An ordinary aspirating needle was employed, about $10 \mathrm{~cm}$. in length, and, with the patient on his side, with thighs flexed on the abdomen and shoulders brought forward, the needle was inserted about $1 \mathrm{~cm}$. beyond the median line, between the third and fourth lumbar vertebræ. This level is crossed by a line connecting the iliac crests.

The only medication any of the patients received, aside from the injections of serum, was an occasional hypodermic injection of morphin combined with hydrobromate of hyoscin, when delirium and restlessness were pronounced. This was not given to all the patients.

The following synopses taken from the clinical charts while the patients were in the hospital show the progress of each case from day to day :

CASE 1.-Patient.-V. H., aged 9, schoolgirl. Referred by Dr. L. R. C. Eberhard. Admitted April 30, 1907, at 9:30 p. m.

History.-Patient first complained of pain in the stomach and legs aching April 28; vomited early in the morning and frequently during the day. She became unconscious about $7 \mathrm{p}$. m. April 28 and was restless and noisy most of the night. The same conditions prevailed on Tuesday morning, when the family noticed retraction of the head and rigidity of the neck. Tuesday evening patient became worse and more restless, throwing and tossing herself about and screaming. Sordes on tongue, lips and teeth.

Condition on Admission.--Temperature 99.6 F., pulse 100, respiration 24. Unconscious; restless and screaming, especially if touched; pupils moderately dilated but fixed; no nystagmus; plantar, patellar and abdominal reflexes absent; Kernig sign positive; herpes labialis; neck rigidity marked and opisthotonus; sphincters intact. 
May 1 and 2: Condition unchanged.

May 2: Temperature 99.6; pulse 100 . Lumbar puncture at $9 \mathrm{a}, \mathrm{m}$. yielded 30 c.c. of opalescent fluid; examination showed diplococci, extra- and intra-cellular.

May 3: Condition practically the same as on preceding day, except for loss of sphincter control.

May 4: Condition no better. On lumbar puncture at 10 a. m. 10 c.c. of fluid withdrawn and 10 c.e. of antiserum injected. Convulsions involving left side and face from 10:40 until midnight; chloroform necessary to control; conscious at 8 p. m.; reflexes absent; Kernig present; neck rigid; nystagmus.

May 5: Temperature normal part of the day; pulse 116 to 120 . Condition improved; called for milk and water; restless at intervals.

May 7: Temperature and pulse normal. Restless and noisy at times.

May 8: Temperature began to rise and reached $103.6 \mathrm{~F}$.

May 9: Complained of frontal headache. On puncture 30 c.c. of fluid withdrawn; 7.5 c.c. of serum injected. Vomited supper. Reflexes absent.

May 10 and 11: Temperature at or near normal all day; child much improved; not very restless.

May 12: Temperature rose to 104.2 ; pulse 140. Restless and noisy in the evening; headache. On puncture at 9 p. m. 60 c.c. of fluid withdrawn; 7.5 c.c. of serum injected; stained specimens failed to show diplococci.

May 13 and 14: Temperature at or near normal; greatly improved.

May 15: Temperature 99 to $104 \mathrm{~F}$; pulse 94 to 136 . Puncture at 8 p. m. yielded 20 c.c. of fluid; no serum injected.

May 16: Child not so well; vomited during the day; severe headache and restless.

May 17: Very irritable; noisy and restless; headache; slight abdominal reflex present.

May 19: Knee and plantar reflexes present for first time; Kernig positive. Rational; irritable, but more quiet than usual; calls for food.

May 20 to 25: No marked change, except for some pain in right leg along course of femoral nerve.

May 25: At 10 a. m. 90 c.c. of fluid withdrawn by puncture; 20 c.c. of serum injected; stain negative. At $8 \mathrm{p}$. m. marked urticaria.

May 26: Temperature normal most of the day; quiet and felt much better; reflexes all present; pain in right leg better; mentality good; involuntary urination. Continued thus for two days.

May 28 to June 3: Temperature ranged between 99 and $103 \mathrm{~F}$.

June 3: Very restless, shouting and erying. At $10 \mathrm{p} . \mathrm{m}$. puncture yielded 75 c.c. of fluid; 15 c.c. of serum injected.

June 4 to 14: Temperature was practically normal; general condition good; rational and moderately quiet most of the time. Reflexes all present and normal; Kernig absent; neck not rigid; perfect function of all parts; no headache; appetite capricious.

June 14: Patient became very restless and noisy. Severe pain in back, leg and abdomen; face flushed; urine and feces involuntary; reflexes all normal; Kernig absent; no neck rigidity.

June 15: At 2 p. m. puncture yielded 75 c.c. of fluid; stain positive; 22.5 c.c. of serum injected. Vomited at midnight.

June 16: Vomited in the morning; refused breakfast; complained somewhat of frontal headache.

June 17: Better to-day; no pain; appetite good.

June 26: Condition good. Reflexes all present and normal; Kernig absent; neck not rigid; no unfavorable sequelæ apparent. Sitting up in bed did not disturb her; less irritable than at any time. Temperature normal from this date on. 


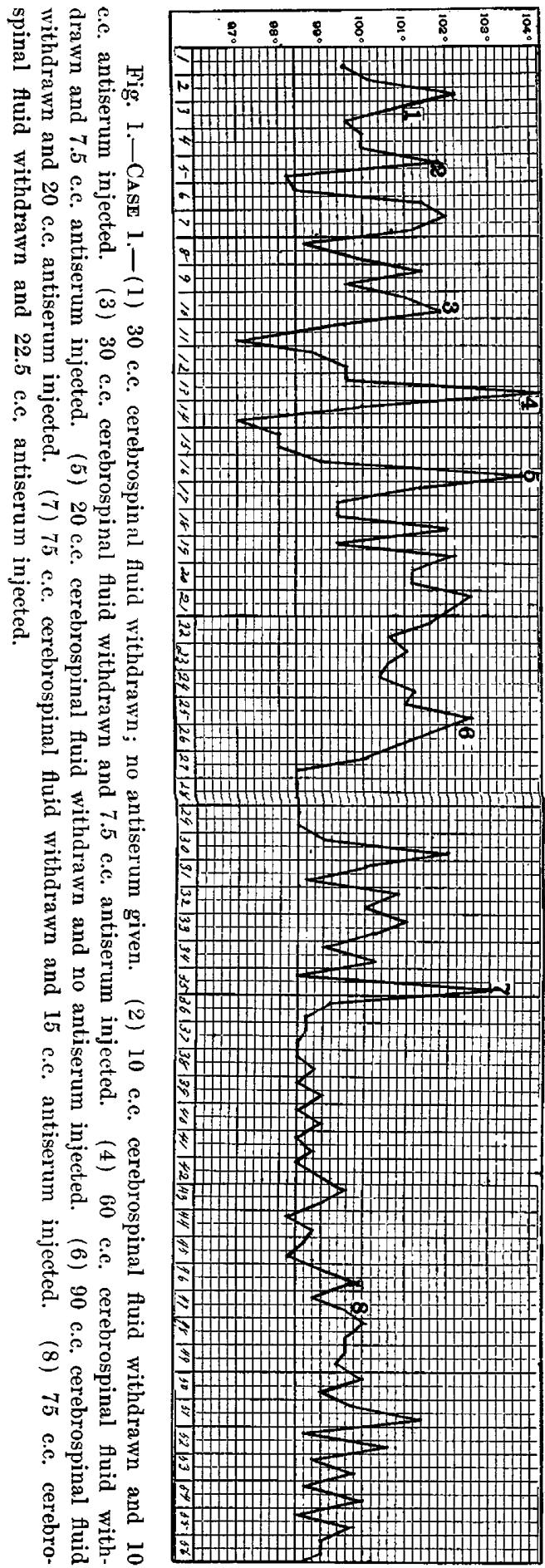


She began to walk, and July 4 commenced to develop a kyphosis; no angular deformity; spine mobile, not painful; only noticeable when standing; involved the whole length of spine. Gymnastics and massage were instituted and used until July 14, when she was put in plaster jacket. The child made a good recovery, and left the hospital Aug. 6, 1907, 96 days after admission. She has been back to the hospital several times since, and is bright mentally and in good condition.

The clinical symptoms in this case were typical, and it would have been almost impossible for one to make an error in diagnosis depending on them alone. This was the first case in which the Flexner antiserum was used. It was brought from Cleveland by Dr. Ladd, who saw the patient in consultation. There was nothing then to govern the dosage in human beings, but the largest dose which had been given up to that time (10 c.c.) was administered May 4, care being taken to inject no more in volume than the fluid withdrawn. This precaution was afterward disregarded as unnecessary, and the dosage was very materially increased. In spite of the precaution, however, the first injection was followed by convulsions. These were not excited by large amounts later on and did not appear spontaneously. It is, therefore, probable that the association was accidental.

As Flexner and Jobling have pointed out in their article which appeared in the January (1908) number of Experimental Medicine, where the first record of the treatment of human beings with this serum is given, it is very difficult to assign a definite effect to the serum in a case so protracted as this. These facts, however, stand out in the history: Within 24 hours following each injection of serum the patient's mental condition was materially improved and the temperature dropped to normal or nearly normal. That improvement was not noticed following a lumbar puncture on May 15, when no serum was injected. Neither was there a fall of temperature nor an improvement in the mental condition when the exacerbations, which occurred periodically throughout the course of the disease, were allowed to go without a lumbar.puncture or an injection of serum.

CASE 2.-Patient.-H. R., aged 17 ; rubber worker. Treated by Dr. George Rankin. Admitted May 9, 1907, at $12 \mathrm{~m}$.

History.-Patient was perfectly well on May 8 and worked, but returned home, not feeling well, complaining of headache; was restless the greater part of the night and did not sleep well; vomited during the night, and became unconscious a few hours before admission to the hospital. He also complained of pain in extremities and cramps in legs.

Condition on Admission.-Temperature $101.6 \mathrm{~F}$; at $11 \mathrm{p} . \mathrm{m}$. temperature was $10 \overline{5} .4$ F. Pulse 90 ; pulseless after 2 p. m. Respiration 40 ; gradually increased to 70 at 10 p. m. Comatose. Pupils: Left contracted, right dilated; did not react to light. Slight nystagmus; plantar, patellar and abdominal reflexes absent; Kernig sign present; no herpes; neck rigid; loss of sphincter control. On 
puncture at 3 p. m. 60 c.c. of opalescent fluid withdrawn; stain positive. A second puncture at $11 \mathrm{p} . \mathrm{m}$. gave 60 c.c. of fluid. The antiserum had to be obtained from Cleveland, and none was received until $11 \mathrm{p}$. $\mathrm{m}$., when 10 c.c. were administered. At that time patient was practically pulseless.

May 10: Temperature at 3 a. m., $106.8 \mathrm{~F}$.; pulseless. Temperature at 5 a. m., $107.2 \mathrm{~F}$. ; Cheyne-Stokes respiration; cyanosed; vomited frequently after $12 \mathrm{~m}$. Died at $5: 42$ a. m.

Case 3.-Patient.-B. S., aged 19; rubber worker. Treated by Dr. W. S. Chase. Admitted May 14, 1907, at 6 a. m.

History.-Patient was well up to May 12, two days before admission to the hospital, when he went to his room complaining of headache. He also had a chill. He was in bed all day May 13; headache worse; vomited and was nauseated; did not eat anything all day. He became unconscious about the time he was moved from his room to start for the hospital.

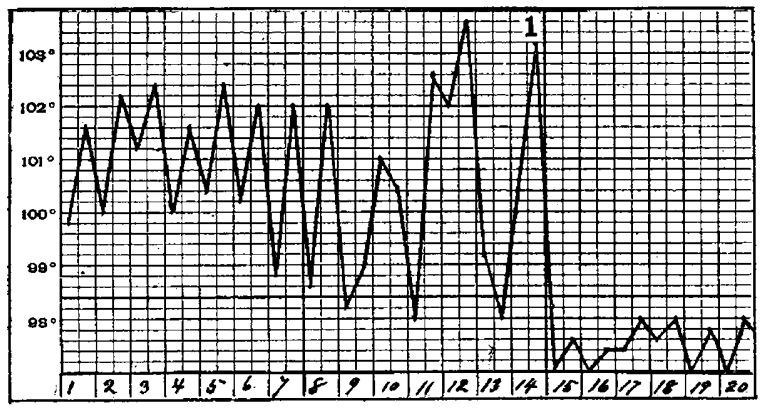

Fig. 2.-CASE 3.-(1) 60 c.c. cerebrospinal fluid withdrawn and 20 c.c. antiserum injected.

Condition on Admission.-Temperature $99 \mathrm{~F}$; at midnight temperature was $101.6 \mathrm{~F}$. Pulse 56 ; 9 a. $\mathrm{m}$. to $12 \mathrm{~m}$. pulseless; midnight, 86 ; markedly cyanosed. Respiration from 32 to 34 and irregular at midnight. Unconscious and wildly delirious; pupils normal; no nystagmus; plantar, patellar and abdominal reflexes absent; Kernig sign positive; no herpes; neck rigid; sphincters intact; urine showed albumin.

May 15: No better. Temperature at 3 p. m., $102.4 \mathrm{~F}$; pulse 80 ; respiration 30. Delirious; neck and spine rigid; talking constantly; gets out of bed; passed a round worm 11 inches long.

May 16: No better. At midnight temperature $102.4 \mathrm{~F}$; pulse 120 ; respiration 26. Involuntary urination. Puncture attempted, but not successful.

May 17: No better. At 6 p. m. temperature $101.6 \mathrm{~F}$; pulse 110 ; respiration 28 . Delirious and restless all day. Coughing; face flushed at times; passed a round worm 9 inches long.

May 18: No better. At 3 p. m. temperature 102.4 F.; pulse 98 ; respiration 28 . Delirious and restless.

May 19: Highest temperature $102.2 \mathrm{~F}$.; pulse 108 and weak; respiration 24. Not quite so restless; involuntary urination. Condition continued practically unchanged until May 22, when temperature was normal all day; passed two round worms. Temperature also normal on May 26, but ranged between 99 and $103.6 \mathrm{~F}$. on May 23, 24, 25 and 27. 
May 27: Physical examination showed neck rigid; slight return of plantar and patellar reflexes; abdominal reflex absent. Lumbar puncture at $10 \mathrm{a} . \mathrm{m}$., under local anesthesia, yielded 60 c.c. of slightly cloudy fluid; 20 c.c. of antiserum injected; stain showed diplococci.

May 28: Temperature normal all day; patient resting and eating well; apparently in good condition.

May 29: Temperature normal all day; neck rigidity less. Patient continued to improve until June 6 , the twenty-fourth day of the disease, when he was able to walk out of the hospital.

Case 4.-Patient.-F. N., aged 32 ; laborer. Treated by Drs. W. S. Chase and Charles E. Held. Admitted May 16, 1907, at 4:30 p. m.

History.-Patient, who was a strong, hearty man, had always been well. He worked on May 15, but complained of severe headache during the latter part of the day and in the evening. He went to his family physician, who prescribed for his pain, but without relief. On the morning of the 16 th he was no better and still complained of severe pain in his head and neck, but had no fever. Morphin, in $1 / 2 \mathrm{gr}$. doses hypodermatically, did not relieve him. A little later he became delirious. Muscular rigidity was marked.

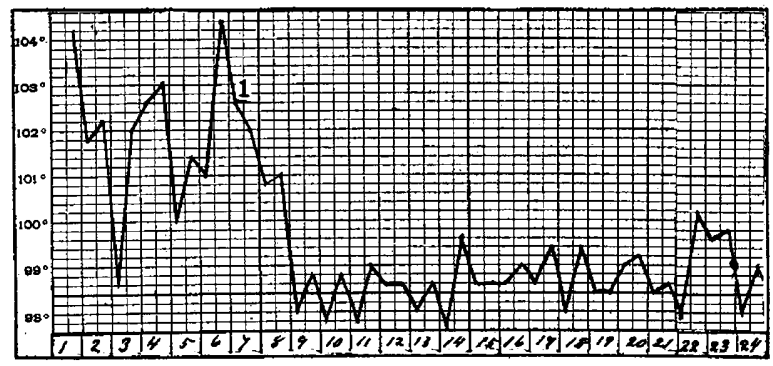

Fig. 3.-CASE 4.-(1) 90 c.e. cerebrospinal fluid withdrawn and 25 c.c. antiserum injected.

Condition on Admission.-Temperature 104.2 F.; pulse 124; respiration 30. Conscious at times only; delirious and restless; pupils normal; plantar, patellar and abdominal reflexes absent; Kernig sign positive; no herpes; no neck rigidity; sphincters intact; no nystagmus.

May 17: Temperature at 6 a. m. 101.8 F.; 9 p. m. 102.2 F. Condition slightly improved; conscious at times; noisy and restless; severe pain in head. Urine showed albumin and casts. Put in restraint. Puncture unsuccessful. Pulse weak at times-128 and 134 .

May 18: Temperature at 6 a. m. $98.6 \mathrm{~F}$; pulse 96 . At $12 \mathrm{~m}$. temperature 102 F.; pulse 100 . Severe pain in head; delirious and noisy.

May 19: Temperature at 6 a. m. $102.6 \mathrm{~F}$; pulse 116 . At 9 p. m. temperature 103 F.; pulse 130. Pain in head severe; delirious and noisy most of the time; hallucinations; respiration 34 to 48 and irregular.

May 20: Temperature at 6 a. m. $100 \mathrm{~F}$; pulse 110 . At 9 p. m. temperature 101.2 F.; pulse 110. Condition the same as on preceding day.

May 21: Temperature at 6 a. m. 101 F.; pulse 106 . At 9 p. m. temperature 104.4 F.; pulse 110 . Condition not much changed; delirious; restless; talking and hiccoughing. 
May 22: Temperature at 6 a, m. $102.6 \mathrm{~F}$; pulse 98 . At $12 \mathrm{~m}$. temperature 102 F.; pulse 98 . On puncture with a long needle 90 c.c. of fluid withdrawn; stain showed diplococci; 25 c.c. of serum injected, Pulse irregular.

May 23: Temperature at 6 a. m. $100.8 \mathrm{~F}$; pulse 96 . At $12 \mathrm{~m}$. temperature 98 F.; pulse 90 . Patient much improved; conscious and resting quietly all day.

May 24: Temperature normal; condition good, except pain in right arm at times. Urine showed trace of albumin; few casts. Appetite good.

May 25: Temperature normal; no pain; felt well; reflexes all present; Kernig absent; neck slightly rigid.

May 26 to 31 : Continued to improve; sat up in bed. Reflexes all present; no pain.

June 1 to 5: Furuncle in right axilla incised. Sat up every day; no pain. Temperature normal.

June 6: Temperature at 6 a. m. $98 \mathrm{~F}$; pulse 98 . At 9 p. m. temperature 100.2 F.; pulse 94. Severe pain in back and legs; unable to move legs; reflexes in legs and feet can not be elicited-rigid; pain and tactile sensation undisturbed.

June 7: Temperature at 6 a. m. $99.6 \mathrm{~F}$; ; pulse 114 . At $12 \mathrm{~m}$. temperature 99.4 F.; pulse 104. Extremities less painful, except when moved; marked tenderness over large nerve trunks; pain in lower jaw on movement.

June 8: Temperature at $6 \mathrm{a} . \mathrm{m}$. $100 \mathrm{~F}$; ; pulse 106 . At $9 \mathrm{p}$. $\mathrm{m}$. temperature 99.4 F.; pulse 104. Felt better; less pain and tenderness not marked. Leucocyte count 22,600

June 9: Temperature normal; much better; moved legs without pain.

June 10 to 15: Neuritis continued to improve. Discharged, feeling well, June 15 , thirty-one days after admission.

Cases 3 and 4 were both marked by a very severe onset. In Case 3 the patient showed extreme cyanosis an hour after admission and was pulseless at the wrist. Diffusible stimulants and oxygen were used freely. These cases are of special interest when compared with some of the others, in that the injections of antiserum were made rather late in the disease. So far as two cases of the same disease can resemble each other they were running the same kind of course. In Case 3 a successful puncture was made on the fifteenth day of the disease. Within 24 hours after an injection of 20 c.c. of antiserum the temperature was normal and the mental condition greatly improved. In Case 4 puncture and injection were performed on the tenth day. Within 24 hours the patient's temperature was normal and he had passed from a period of wild delirium to one of complete consciousness and quiet. It is noteworthy to observe, also, that both patients, save for the neuritis in Case 4, made speedy and uneventful recoveries.

CASE 5.-Patient.-B. K., aged 15; factory girl. Treated by Dr. L. R. C. Eberhard. Admitted May 16, 1907, at 9 a. $\mathrm{m}$.

History.-Patient had some headache May 15. It was not very severe, but she noticed that she was more nervous than usual. She slept well that night and was conscious on the morning of May 16. Still complained of headache, which was more severe than on the day before. She refused breakfast. Her mother gave her an enema, thinking it would relieve her pain. After this the mother went downstairs and returned in about twenty minutes to find the patient unconscious. The family physician was called and found the patient throwing her- 
self around and tossing about on the bed. Rigidity was very marked. She was sent to the hospital at once.

Condition on Admission.-Temperature 103.6 F.; pulse 84 and irregular; respiration 34 ; comatose; pupils dilated, but reacted; nystagmus; plantar, patel. lar and abdominal reflexes absent; Kernig sign positive; no herpes; neck rigid; sphincters intact; urine showed albumin. Lumbar puncture gave 45 c.c. of opalescent fluid; diplococei present; 15 c.c. of antiserum injected.

May 17: Temperature at 6 a. m. $99.8 \mathrm{~F}$; pulse 70 ; respiration 24 . At $12 \mathrm{~m}$. temperature $102.6 \mathrm{~F}$.; pulse 100 ; respiration 26 . Conscious; noisy and restless at times and complained of severe headache.

May 18: Temperature at 6 a. m. $101.6 \mathrm{~F}$; pulse 94 ; respiration 20 . At $12 \mathrm{~m}$. temperature 100.2 F.; pulse 82 ; respiration 24. Semiconscious; knee and plantar reflexes present; abdominal reflex absent; Kernig sign present; pain in head severe; noisy at times; involuntary urination.

May 19: Temperature at 6 a. $\mathrm{m} .100 .8 \mathrm{~F}$; pulse 90 ; respiration 20 . At $12 \mathrm{~m}$. temperature 102 F.; pulse 96 ; respiration 22 . Semiconscious, but rational when spoken to; herpes labialis appearing; knee and plantar reflexes diminished; abdominal reflex absent; internal squint of both eyes-more pronounced in right. On puncture there was withdrawn 4.5 c.c. of cloudy fluid; 5 c.c. of serum injected.

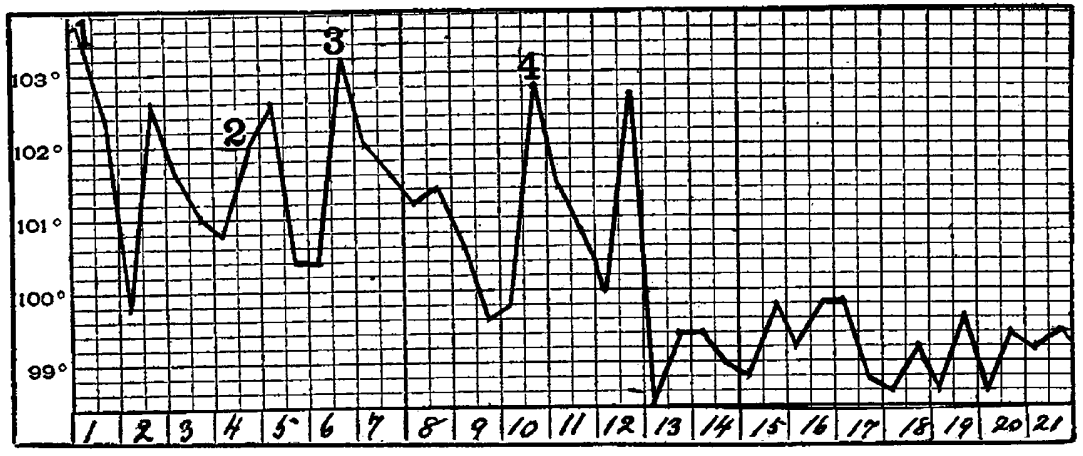

Fig. 4.-CASE 5.-(1) 45 c.c. cerebrospinal fluid withdrawn and 15 c.c. anti serum injected. (2) 45 c.c. cerebrospinal fluid withdrawn and 5 c.c. antiserum injected. (3) 4 c.c. cerebrospinal fluid withdrawn and 3.5 c.c. antiserum injected. (4) 75 c.c. cerebrospinal fluid withdrawn and 20 c.c. antiserum injected.

May 20: Temperature 100.8 to $102.6 \mathrm{~F}$; pulse 106 to 120 . About same condition as on day preceding; strabismus not quite so marked; rational when spoken to; unable to move arms; headache severe.

May 21: Temperature in the morning 103.2 F. Puncture yielded 4 c.c. of fluid; 3.5 e.e. of serum injected; stain positive.

May 22: Very irritable; headache severe; pain all over on being moved; herpes on ear; squint not so marked; pupils dilated, react; knee and plantar reflexes present; transient hyperemic areas on abdomen.

May 23: Restless and noisy; severe pain in left leg; unable to move left arm and leg; headache constant.

May 24: Temperature at 6 a. m. $100.6 \mathrm{~F}$; pulse 120 . At $12 \mathrm{~m}$. temperature 102 F.; pulse 100. Semiconscious; irritable; pain in head, left arm and leg; right arm all right. 
May 25: Condition same as on preceding day, except decubitus of left heel and elbow: tactile and pain sensations normal on left side; 75 c.e. of fluid withdrawn by puncture and 20 c.e. of serum injected; stain failed to show diplococei ; involuntary urination and bowel movements.

May 26: Temperature at 6 a. m. $101.4 \mathrm{~F}$; pulse 108. At $12 \mathrm{~m}$. temperature 100.6 F.; pulse 120 . Patient better; complains of pain in head at times and is restless and noisy; rational when spoken to; reflexes same as on preceding day.

May 27: Temperature at 6 a. m. $100 \mathrm{~F}$; ; pulse 104. At $12 \mathrm{~m}$. temperature 102.6 F.; respiration 26. Reflexes all present; conscious; neck rigid; Kernig sign present; pain in head, arms and legs on movement.

May 28: Temperature 98.4 to 99.4 F. Urticaria on knees and elbows.

May 30: Patient improved. Some headache at times; urine normal; no involuntary urination; knee and plantar reflexes normal; abdominal reflex absent; Kernig sign present; neck less rigid; left arm and leg well; sphincters normal. From this day patient gradually improved, highest rectal temperature being $99.8 \mathrm{~F}$. Her appetite was good and she was free from pain, but she did not gain strength rapidly.

June 8: Kernig sign absent; neck not so rigid; no pain anywhere; felt well, and appetite excellent. She had normal temperature from this date until date of discharge, June 27 , being 41 days after admission to the hospital. She was perfectly well in every way, except not as strong as formerly. Patient came to the hospital early in September, feeling all right in every way.

In the light of our present knowledge, it is apparent that the first three injections in this case were entirely too small, especially the second and third. It is uncertain, of course, whether larger doses at these times would have affected the course of the disease, but they should certainly have been given.

CASE 6.-Patient.-Z. H., female, aged 17 ; rubber worker. Treated by Dr. W. S. Chase. Aơmitted May 19, 1907 , at 6 p. m.

II istory.-Patient had been well, and worked until Saturday noon preceding. She complained somewhat of soreness and aching in the back and legs. At $2 \mathrm{a} . \mathrm{m}$. May 19 she complained of headache and became unconscious about $3 \mathrm{a} . \mathrm{m}$. She was first seen by a physician at 7 a. $\mathrm{m}$. and was reported to have some fever. During the early morning and tnroughout the day she vomited several times and was unconscious and very restless, continually tossing about, rolling her eyes and head and holding herself in tonic contractures.

Condition on Admission.-Temperature at 6 p. m. 101.6 F.; 99 F. at mid night. Pulse at 6 p. m. $104 ; 80$ at midnight. Respiration at 6 p. m. 28 ; 18 at midnight. Comatose; pupils equal, moderately dilated and reacted to light; nystagmus; plantar and patellar reflexes present; abdominal absent; Kernig sign present; no herpes; neck rigid; control of anal sphincter lost. Lumbar puncture gave 45 c.c. of opalescent fluid; diplococci present; 15 c.c. of antiserum injected. May 20: Temperature normal; patient conscious; talked rationally to members of her family at 3 a. $\mathrm{m}$.; some frontal headache.

May 21: Temperature normal; mentality not quite so good; involuntary urination and bowel movements; herpes appearing on lips; hyperemic areas on abdomen. At 9 p. m. 60 c.c. of fluid withdrawn by lumbar puncture; diplococei present; 7.5 c.c. of antiserum injected.

May 22: Temperature at midnight $99.2 \mathrm{~F}$; p pulse 92 ; respiration 18 . Patient in good condition. Died suddenly at 12:30 a. m. No postmortem permitted. 
It will be noted from the history that this patient became unconscious at 3 a. m. on May 19 . She was seen by a physician at $y$ a. m. and admitted to the hospital at $6 \mathrm{p}$. m. on the same day. She was given 15 c.c. of the antiserum about an hour after admission. At $3 \mathrm{a} . \mathrm{m}$. the next day her temperature was normal. She had passed from a deeply comatose state to one of perfect consciousness and conversed freely with members of her family. The change was so marked and so impressed the members of the family that they thought a miracle had been performed.

The second injection was given on the evening of May 21, not because her general condition did not seem excellent, but because she was slightly duller mentally than on the day before. She was reported in good condition at 11:30 p. m. An hour later the attending nurse heard her breathing heavily, and in five minutes she was dead. An autopsy was not permitted, and death must be put down to one of those accidents liable to occur at any time during the course of a severe infection. We can not connect in any way her sudden death with the second puncture. She took the anesthetic well and was in good condition after coming out from under its influence.

Case 7.-Patient.-H. S., aged 14; school boy. Treated by Dr. A. F. Sippy. Admitted May 22, 1907, at 8 p. m.

History.-Patient had been well and first complained of headache the evening of May 21. On the morning of May 22 he had vomited twice and complained of severe pain in the head. His temperature was $101 \mathrm{~F}$; pulse normal. At $5 \mathrm{p.}$. he became delirious, with pupils dilated and fixed, but no neck rigidity.

Condition on Admission.-Temperature 104 F.; pulse 120; respiration 44; unconscious; comatose; wildly delirious and noisy; pupils dilated and fixed; no nystagmus; plantar, patellar and abdominal reflexes absent; Kernig sign present; no herpes; neck slightly rigid; sphineters intact. On puncture 75 c.c. of opalescent fluid obtained; diplococci present; 15 c.c. of antiserum injected.

May 23: Improved; semiconscious; temperature $100 \mathrm{~F}$; pulse 80 ; respiration

26 ; restless and noisy at times; rational at times and complained of severe headache.

May 24: Temperature at 6 a. m. 101.8 F.; 9 p. m. 99.8 F. Rational all day; vomited once; severe headache at night.

May 25: Temperature at 9 a. m. 102 F.; pulse 66 ; respiration 26. On puncture 75 c.c. of fluid obtained; 20 c.c. of serum injected. Rational all day. Temperature at 9 p. m. 98.8 F.; pulse 68 ; respiration 24 .

May 26: Improved; temperature normal all day; pain in head less; reflexes all present; neck rigidity less.

May 27: Improved; temperature at 7 p. m. $98 \mathrm{~F}$; resting well; some pain in head.

May 28: Temperature at 7 p. m. 99.8 F.; resting well; no pain; neck slightly rigid.

May 29: Temperature at 3 p. m. 100.2 F.; normal at $12 \mathrm{~m}$; no pain.

May 30: Temperature $100 \mathrm{~F}$. at $12 \mathrm{~m} ., 3 \mathrm{p} . \mathrm{m}$. and $6 \mathrm{p} . \mathrm{m}$.; normal at $9 \mathrm{p} . \mathrm{m}$. ; appetite better; no pain.

May 31: Temperature at 6 p. m. 99.8 F. 
June 1: Temperature at 3 p. m. 99.2 F.

June 2: From this day on temperature was normal.

June 8: On this day, 18 days after admission, patient sat up in bed three hours. Discharged from hospital.

This case, while not of the fulminating type, was certainly severe. Any one seeing the boy at the time of admission to the hospital would surely have been justified in giving a very grave prognosis. This being the seventh case in our series, the attending physician became bold enough to give the boy 15 c.c. of antiserum at the first injection. The symptoms

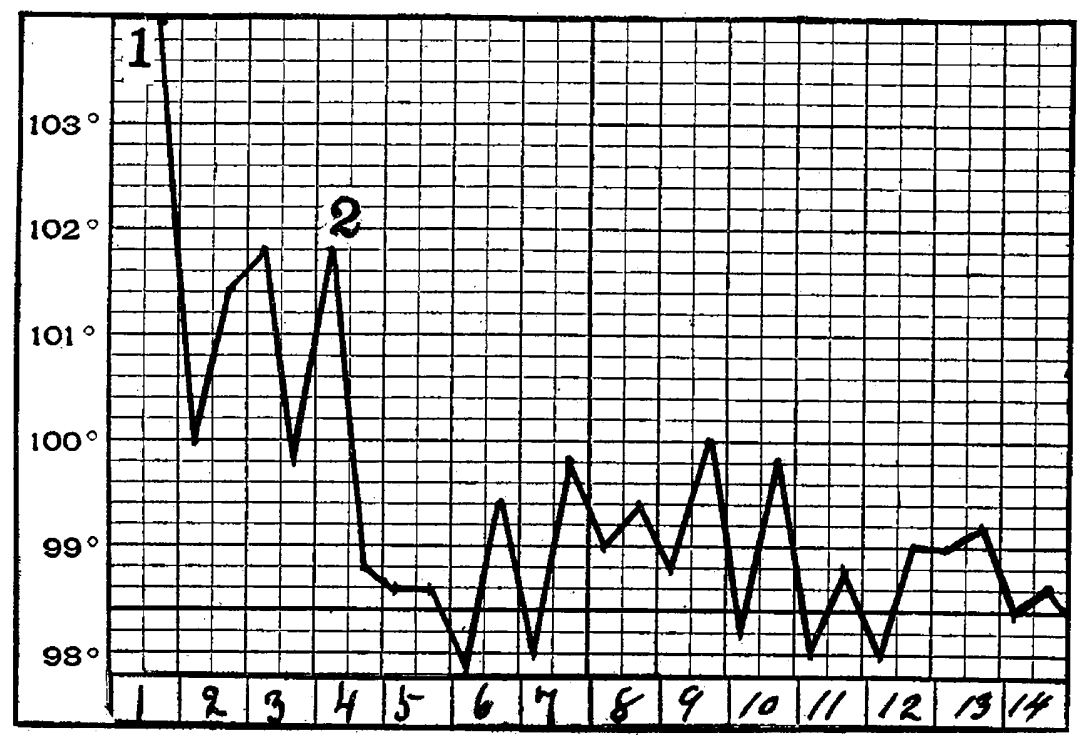

Fig. 5.-CASE $7 .-$ (1) 75 c.c. cerebrospinal fluid withdrawn and 15 c.c. of antiserum injected. (2) 75 c.c. cerebrospinal fluid withdrawn and 20 c.c. antiserum injected.

did not abate so rapidly in this case as in some of the others, and on the fourth day of illness the temperature rose to $103 \mathrm{~F}$. A puncture was made and 75 c.c. of spinal fluid withdrawn. It was then decided to give 20 c.c. of antiserum. In ten hours his temperature was normal and he made an uninterrupted recovery.

Case 8.-Patient.-R. M., aged 17 ; electrician. Treated by Dr. W. S. Chase. Admitted May 25, 1907, at 1 p. m.

History.-Patient, who was an employe of a local rubber company, quit work at noon on May 24 because he did not feel well. He went home, but did not go to bed; refused supper, and later in the evening vomited. He went to bed early, complaining of headache, and was restless the greater part of the night. His headache was worse in the morning. He refused breakfast, and vomited again. About 10 a. $\mathrm{m}$. he became unconscious. 
Condition on Admission.-Temperature 105.2 F.; pulse 142; respiration 20; comatose-violent; pupils slightly contracted and reacted to light; no nystagmus: plantar reflex present, patellar slightly exaggerated, abdominal absent; Kernig sign present; no herpes; neck rigid and painful to move; sphincters intact. On puncture 15 c.c. opalescent fluid withdrawn; diplococci present; 15 c.c. serum injected.

May 26: Patient worse. Temperature 102.2 F.; pulse 100 ; respiration 6 ; cyanosed; puncture and withdrawal of 15 c.e. of fluid, stain of which showed diplococci. Died shortly after midnight.

CAsE 9.-Patient.-G. G., aged $1 \overline{7}$; school girl. Treated by Drs. G. A. Theiss and A. F. Sippy. Admitted June 9, 1907, at 7 p. m.

History.--Patient was well until 24 hours before admission to the hospital, when she began to complain of malaise and headache. She vomited during the night and became unconscious at 2:30 p. m. June 9, just $4 \frac{1}{2}$ hours before admission to the hospital.

Condition on Admission.-Temperature 98.4 F.; pulse 108; respiration 20. Unconscious; comatose and very restless; pupils normal; no nystagmus; plantar, patellar and abdominal reflexes absent; Kernig sign positive; no herpes; neck

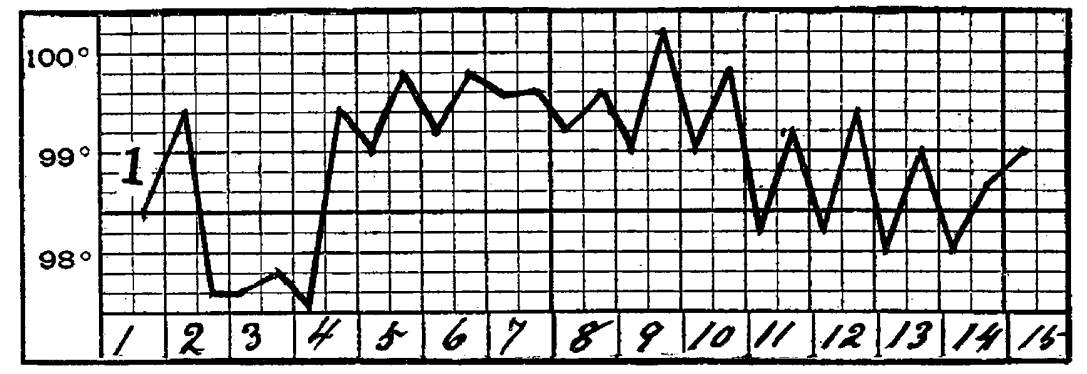

Fig. 6.-CASE 9.-(1) 90 c.c. cerebrospinal fluid withdrawn and 22.5 c.c. antiserum injected.

rigid; sphincters intact. Puncture yielded 90 c.c. of opalescent fluid; stain positive; 22.5 c.c. of antiserum injected.

June 10: Temperature at 6 a. m. 97.2 F.; pulse 102. Temperature subnormal all day; rational at times; slight opisthotonus and nystagmus. It was necessary to use a catheter.

June 11: Temperature subnormal all day; conscious and rational; abdominal and plantar reflexes present; patellar reflex absent; Kernig sign marked; neck rigid; herpes labialis; no pain.

June 12: Highest temperature 99.6 F.; pulse 84. Mental condition excellent; spoke of pain in head only when asked; reflexes all present; Kernig sign present; neck rigid and painful on movement; got out of bed and went to closet alone. Patient gradualıy improved from the fourth to the twelfth day of the disease. Reflexes all returned. Neck rigidity disappeared. Kernig sign disappeared. Appetite improved. Free from pain most of the time.

June 21: Temperature normal; sat up in chair; ate and slept well; no sequelæ. June 23: Discharged, 15 days after admission.

The onset in this case was not very severe, although within 24 hours the patient was in a comatose condition and opisthotonus was marked. 
All the reflexes were lost. Despite these symptoms and the presence of large numbers of the Diplococcus intracellularis, the patient's temperature was normal and the respirations were but slightly accelerated.

CASE 10.-Patient.-J. A. S., aged 24; postman. Treated by Dr. George Rankin. Admitted June 24, 1907, at 11 a. m.

History._-Patient had been well, and worked until the day before his admission to the hospital. On that day he attended a picnic and was exposed to the hot sun. He went home in the evening with headache and feeling ill. This he attributed to the effects of the sun. He did not sleep well and was restless, complained more of headache, and became unconscious about 5 a. m. July 24.

Condition on Admission.-Temperature 103.8 F.; pulse 70 ; respiration 36 ; comatose and very restless; face flushed; got out of bed; pupils moderately dilated, respond to light; plantar, patellar and abdominal reflexes absent; Kernig sign positive; no herpes; neck moderately rigid; involuntary urination and bowel movements; no nystagmus. Puncture yielded 90 c.c. of fluid; stain positive; 22.5 c.c. of serum injected.

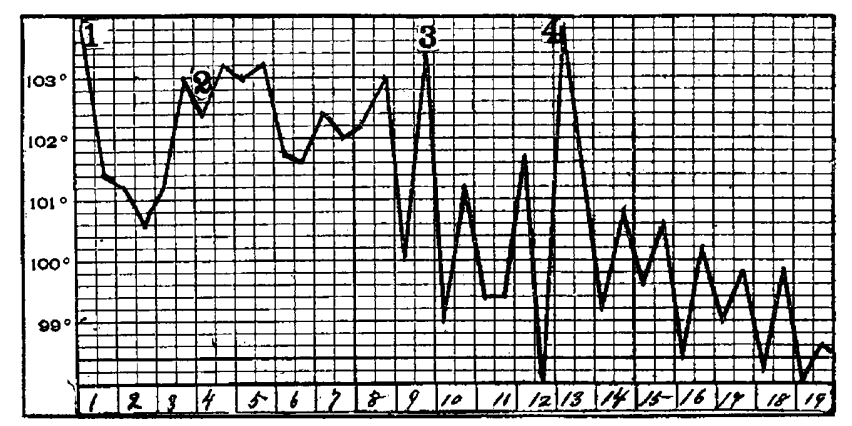

Fig. 7.-CASE 10.-(1) 90 c.c. cerebrospinal fluid withdrawn and 22.5 c.c. antiserum injected. (2) 45 c.e. cerebrospinal fluid withdrawn and 22.5 c.c. antiserum injected. (3) 60 c.c. cerebrospinal fluid withdrawn and 30 c.c. antiserum injected. (4) 45 c.c. cerebrospinal fluid withdrawn and 30 c.c. antiserum injected.

June 25: Temperature at 6 a. m. 101.2 F.; pulse 60 . At $12 \mathrm{~m}$. temperature 100.6 F.; pulse 64. Semiconscious; responds when spoken to, but mutters unintelligibly when attempting to form sentences; restless at times; knee reflex absent; plantar and abdominal reflexes present; neck more rigid; Kernig sign present; pupils equal, dilated and respond to light.

June 26: Temperature at 6 a. $\mathrm{m}$. $101.2 \mathrm{~F}$; pulse 64 . At $12 \mathrm{~m}$. temperature 103 F.; pulse 72 . Conscious and rational; neck less rigid; some headache.

June 27: Temperature at 6 a. $\mathrm{m}$. $102.4 \mathrm{~F}$; ; pulse 70 . At $12 \mathrm{~m}$. temperature 103.2 F.; pulse 92 . Herpes labialis appearing. At 2 a. m. puncture gave 45 c.c. of fluid; 22.5 c.c. of serum administered. At 9 a. m. rational; singing and talking with other patients; reflexes improved; Kernig sign present; neck rigid; involuntary urination.

June 28: Temperature at 6 a. m. $103 \mathrm{~F}$; pulse 74 . At $12 \mathrm{~m}$. temperature 102 F. : pulse 80 . Mental condition good; some pain in back of neck. 
June 29: Temperature at 6 a. m. 101.8 F.; pulse 80 . At $12 \mathrm{~m}$. temperature 101.2 F.; pulse 74. Same condition as on preceding day. Pain in back of neck and head.

June 30: Temperature at 6 a. m. 102.4 F.; pulse 72 . At $12 \mathrm{~m}$. temperature $101.6 \mathrm{~F}$; pulse 80 . Condition good; reffexes all present; Kernig sign present; neck rigid and painful on movement; some headache; herpes labialis extensive and painful.

July 2: Temperature at 6 a. m. $100.4 \mathrm{~F}$; pulse 70 . At 9 p. m. temperature $103.6 \mathrm{~F}$. ; pulse 80 . Condition the same as on preceding day. At $9 \mathrm{p} . \mathrm{m}$. puncture gave 60 c.c. of fluid; 30 c.c. of serum injected.

July 3: Temperature at 6 a. m. $99 \mathrm{~F}$; pulse 70 . At $12 \mathrm{~m}$. temperature $100 \mathrm{~F}$; pulse 74 . Condition improved; feels better.

July 4 to 6: No essential change, except for pain in testicles; not red or swollen, but very painful to touch.

July 6: Puncture yielded 45 c.c. of slightly cloudy fluid; 30 c.c. of serum injected; stain positive.

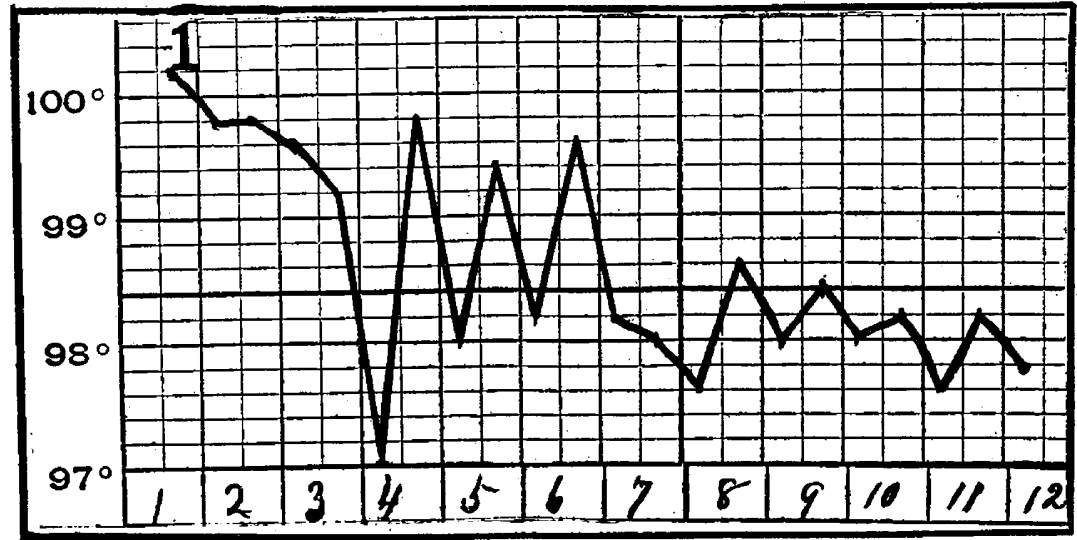

Fig. 8.-CASE 11.-(1) 75 c.c. of cerebrospinal fluid withdrawn and 25 c.c. antiserum injected.

July 7 : Temperature at 6 a. m. $99.2 \mathrm{~F}$; pulse 72 . At $12 \mathrm{~m}$. temperature 99.8 F.; pulse 84 . Patient feels well; no pain.

July 8: Temperature at 6 a. m. $99.6 \mathrm{~F}$; pulse 80 . At $12 \mathrm{~m}$. temperature 99.2 F.; pulse 70. No pain; appetite excellent.

July 9: Temperature normal; no pain; no neck rigidity. Temperature remained normal from this day on.

July 14: Sat up in bed. Patient continued to improve, grew stronger, walked about the ward, and was discharged July 24 at 3 p. m., 31 days after admission to the hospital. Reported since that he can not stand the hot sun; it causes him intense headache.

CASE 11.-Patient.-E. R., aged 20; machinist. Treated by Drs. C. T. Hill and J. H. Seiler. Admitted June 30, 1907, at 9:50 p. m.

History.-Patient worked all day Saturday preceding, and went to church Sunday morning feeling well. He came home and did not eat much dinner, having a little headache. After dinner he lay down in the yard in the shade for a 
while; then went upstairs to bed. He was found about $3 \mathrm{p} . \mathrm{m}$. lying on the floor in an unconscious state, rolling about at times and throwing his hands to his head.

Condition on Admission.-Temperature 100.2 F.; pulse 80; respiration 18; unconscious; comatose; restless, moaning and throwing his hands to his head; pupils normal; no nystagmus; plantar and patellar reflexes exaggerated, abdominal absent; Kernig sign present; no herpes; neck slightly rigid and painful on movement; sphincters intact. Puncture yielded 75 c.c. of opalescent fluid; stain showed diplococei; 25 c.c. of serum injected.

July 1: Temperature at 6 a. m. $99.8 \mathrm{~F}$; pulse 80 ; slight convulsion, lasting 20 minutes, at 3 a. $\mathrm{m}$; ; vomited several times during the day. Temperature at 12 m. $100 \mathrm{~F}$. ; pulse 60 ; restless and moaning.

July 2: Temperature at 6 a. m. 99.6 F.; pulse 54 . Much improved; rational; reflexes all present; Kernig sign less marked; neck still rigid, but not so painful on movement; some pain in head; mentality good. Temperature at $6 \mathrm{p} . \mathrm{m}$. $99.2 \mathrm{~F}$; pulse 68 .

July 3: Temperature at 6 a. m. 97; pulse 62 . Condition excellent; only complaint is pain over eyes. Temperature normal most of the day; at 6 p. m. 99.8 F.; pulse 72 .

July 4: On this the fourth day of the disease temperature was normal and remained so. Reflexes all present and normal.

July 9: Patient sat up in a chair.

July 11: On this day, 12 days after admission, patient was discharged, walking to the street car.

The onset here was abrupt and severe. This case was received at the hospital earlier than any of the others. From the appearance of premonitory symptoms to the time of the first injection only 12 hours had elapsed. He then received 25 c.c. of serum. Following this early large dose, his symptoms rapidly abated.

CASE 12.-Patient.-H. B., aged 6; schoolgirl. Treated by Dr. Charles E. Norris. Admitted Sept. 11, 1907, at 1:30 p. m.

History.-Patient attended school Monday, Tuesday and Wednesday, but stayed at home Thursday with a sprained ankle. Friday she suffered with a severe headache; had paroxysms of pain, and put her hands to her head and cried out. She then passed into a semi-comatose state. This continued until Tuesday morning about 11 o'clock, when she went into a comatose state and so remained until the time of her admission to the hospital. On admission her head was not drawn back, but neck was rigid and on attempting to move it her facial expression was that of a person in pain. The same was true in eliciting Kernig's sign. Abdomen was retracted; tâche cérébrale present; tongue and mouth dry, and sordes on tongue and teeth. Patient had been treated from Friday until Tuesday for intestinal trouble, and on Wednesday the death sentence was pronounced.

Condition on Admission.-Temperature 102.6 F.; midnight, 103.4 F. Pulse 132 ; midnight, 138. Respiration 22; midnight, 28 . Unconscious; comatose; pupils moderately dilated, but reacted to light; plantar, patellar and abdominal reflexes absent; Kernig sign present; no herpes; neck rigid; sphincter control lost; nystagmus at times. On puncture 35 c.c. of clear fluid was withdrawn, and 30 c.c. of serum injected; stain positive. It was feared that the child would die while the puncture and injection were being made. No anesthetic.

September 12: Temperature at 6 a. m. $103.4 \mathrm{~F}$. ; pulse 124. Temperature at 12 m. 101.4 F.; pulse 114. Semi-conscious; respiration more regular; complete 
paralysis of legs and right arm, but moved left arm at times. Puncture at 1 p. m. gave 35 c.c. of fluid; 30 c.c. of serum injected; stain positive. Involuntary urination and bowel movements; reflexes absent; neck rigid.

September 13: Temperature at 6 a. m. $101 \mathrm{~F}$; pulse 110. Temperature at 9 p. m. $100.4 \mathrm{~F}$; pulse 94 . Improved; responds when spoken to; takes nourishment; reflexes all absent. Puncture at $10 \mathrm{p}$. $\mathrm{m}$. gave 30 c.c. of fluid; 30 c.c. of serum injected. Neck rigid.

September 15: Temperature 99.8 to 100.4 F.; pulse 96 . Improved; mental condition better; pronates and supinates left arm; complains of pain on being moved.

September 17: Condition about the same. Neck not so rigid; moves it laterally. Pain on pressure over muscles and large nerve trunks; reflexes absent.

September 19: Somewhat better; enjoys being played with; moves both arms somewhat, also toes; plantar reflex present for first time, others absent; nerves respond to Faradic current; tactile, thermal, and pain senses all present.

September 21: General condition better; plays with doll and laughs. Puncture at $9: 30$ p. m. gave 40 c.c. of fluid; 30 c.c. of serum injected.

September 22: Temperature at 6 a. m. $101 \mathrm{~F}$; pulse 108 . Temperature at $12 \mathrm{~m}$. $99.2 \mathrm{~F}$; pulse 112 . Condition about the same as on preceding day; somewhat irritable; no rigidity of neck; muscular soreness.

September 23: Moves hands better; abdominal reflex on right side; irritable; small ulcer above inion; plantar reflex present at all times; others absent; no neck rigidity.

September 24: Urticaria over extensor surfaces.

September 26: No change, except for adenopathy on right side of neck.

September 28: Temperature at 6 a. m. 100.4 F.; pulse 116. Temperature at 6 p. m. 102 F.; pulse 136. Adenopathy more marked; moves arms and toes freely and left knee slightly.

September 29: Adenopathy on both sides; sphincter control improved; reflexes the same.

October 4: Urticaria gone; adenopathy more marked. Puncture at 9 p. m. gave 75 c.c. of fluid; 25 c.c. of serum injected. Right side of neck incised and drained; reflexes the same.

October 5: Temperature at 6 a. m., 102.8; pulse, 134 . Temperature at 9 p. m., 102.4 F.; pulse, 140. Condition about the same as on preceding day; pain in neck.

October 7: Temperature at 6 a. m., $100.6 \mathrm{~F}$; pulse, 110 . Temperature at 9 p. m., $100.6 \mathrm{~F}$; pulse, 108. Condition improved; adenopathy improved; no more involuntary urination or bowel movements.

October 11: Condition same, as on preceding three days. Puncture at $9 \mathrm{p} . \mathrm{m}$. yielded 65 c.c. of fluid; 15 c.c. of serum injected.

October 12: Internal squint of right eye; pain on being moved; adenopathy disappeared.

October 13: Squint in both eyes; pupils equal and react; erythema on back.

October 15 to 23 : Temperature but slightly above normal; moved arms and fingers a little better each day.

October 23: From this day on the child had normal temperature and pulse, was not so irritable, and ceased to have pain on being moved. Her mental condition was good-her people say as good as it ever was. The paralysis improved considerably before she was discharged, so that she was able to feed herself; could invert and evert both feet; moved the left knee very well, and also the toes. She enjoyed sitting up in a wheel chair. She gained about 15 pounds in weight while at the hospital, and her appetite and digestion were good all the time. Reflexes did not improve. On the date of her discharge, plantar reflex was 


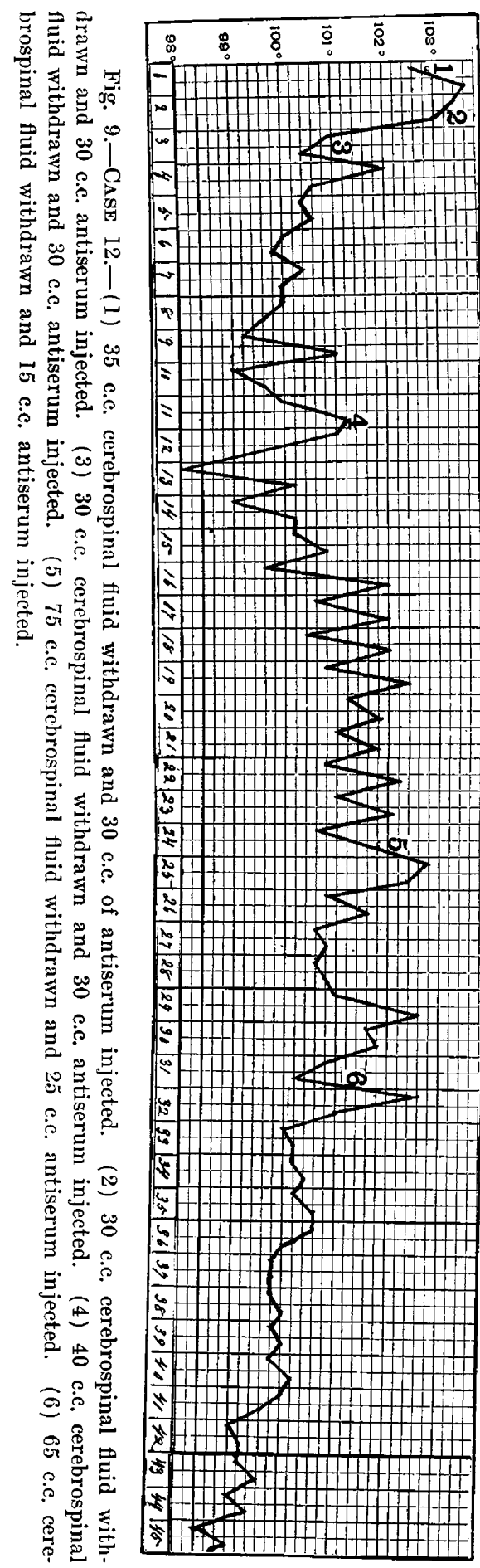


present, patellar absent, abdominal present on right side only; Kernig sign absent. She still had some internal squint of both eyes, but it had improved; pupils equal and reacted to light and accommodation. She knew her letters and could see to read them and also to count. She was discharged Nov. 13, 1907, the sixty-fourth day after entering the hospital.

This case ran a very severe and protracted course. The first puncture was made without an anesthetic of any kind, and 30 c.c. of serum were injected. As stated, the child collapsed under this procedure, and it was thought for a time that she would not revive. On the second and third days thereafter punctures were made and antiserum injected. She was semi-conscious on the second day, rational and able to take nourishment on the third day. It will be noted that a small decubitus ulcer developed over the inion on the thirteenth day, and it was undoubtedly from this source that there resulted an infection of the cervical lymph glands noted in the history. Part of the fever and irritability from the sixteenth to the twenty-sixth day might certainly be attributed to this infection.

\begin{tabular}{|c|c|c|c|c|c|c|c|}
\hline 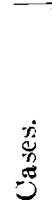 & 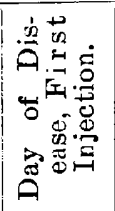 & 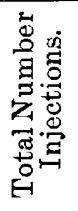 & 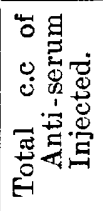 & 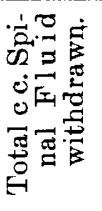 & $\begin{array}{c}\text { Complica- } \\
\text { tion. }\end{array}$ & Sequelæ. & Results. \\
\hline 1. & 7 & 6 & 82.5 & 390 & None. . . & Kyphosis. & Recovery by lysis \\
\hline 2 & 2 & 1 & 10.0 & 120 & $\cdot \cdot$ & . & Death \\
\hline 3 & 15 & 1 & 20.0 & 60 & Nephritis. . & None. . . . & Recovery by crisis. \\
\hline 4 & 10 & 1 & 25.0 & 90 & $\begin{array}{l}\text { Nephritis } \\
\text { neuritis, } \\
\text { furuncles. }\end{array}$ & None. .... & Recovery by crisis. \\
\hline 5 & 2 & 4 & 43.5 & 169 & Neuritis. . . & None. . . . & Recovery by lysis. \\
\hline 6 & 2 & 2 & 22.5 & 105 & . . & . & Death. \\
\hline 7 & 1 & 2 & 35.0 & 150 & None. . . . & None. . . & Recovery by crisis. \\
\hline 8 & 2 & 1 & 15.0 & 30 & $\ldots$ & $\ldots$ & Death. \\
\hline 9 & 1 & 1 & 22.5 & 90 & None. & None. . . & Recovery by crisis. \\
\hline 10 & 2 & 4 & 105.0 & 240 & None. . . . & $\begin{array}{l}\text { Headache in } \\
\text { sun. }\end{array}$ & Recovery by lysis. \\
\hline 11 & 1 & 1 & 25.0 & 75 & None. & None.... . & Recovery by crisis. \\
\hline 12 & 6 & 6 & 160.0 & 275 & 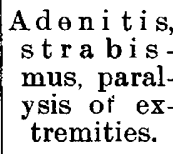 & $\begin{array}{l}\text { Partial par- } \\
\text { alysis of } \\
\text { extremi- } \\
\text { ties. }\end{array}$ & Recovery by lysis. \\
\hline
\end{tabular}




\section{CONCLUSIONS.}

Although the figures given above record 75 per cent. of recoveries in cases treated with the antiserum, we feel that it is really unfair to put Case 2 in the failure column, for the serum was not used until some time after the patient had become pulseless. In Case 6 the improvement following the first injection was as marked and as satisfactory as in any of those which went on to recovery. Death came suddenly and without a warning. The remaining case which resulted fatally was of the most pronounced fulminating type.

While we have not the clinical records of the cases outside the hospital which did not receive the antiserum, all of which died but one, we certainly have the right to assume that, occurring in the same epidemic, the difference in mortality in these cases as compared with those that received the antiserum was not due simply to their having been of much greater average severity. 